\title{
A new model for bioentrepreneurship?
}

\author{
Stephen M. Edgington
}

When we published the first Bioentrepreneurship supplement in May 1998, we asked a select group of biotechnology and pharmaceutical executives, investment bankers, venture capitalists, consultants, and lawyers what advice they would give to someone who was building a biotechnology company. The demand for the advice they offered was so overwhelming that we soon discovered we had not printed enough copies to fulfill all requests.

This enthusiastic response prompted us to develop a second Bioentrepreneurship supplement, calling once again on the biotechnology industry's deep pool of talent for advice and comment. We gave our authors the option of addressing a theme that had emerged in the first supplement-that the biotechnology industry needed to consolidate in much the same way the pharmaceutical industry has done and continues to do. What is clear from the contributions to this supplement is that the biotechnology industry is undergoing a major transition-perhaps the greatest since it began - and that for those willing to adapt, there are many opportunities up ahead.

\section{An industry in transition}

Our authors were quick to point out that a radical restructuring of the environment in which the biotechnology industry has been nurtured thus far is taking place. In one sense, the industry has outgrown the niche it has so comfortably inhabited for nearly a quarter of a century, and needs to adapt if it is to successfully compete for the resources it needs to fully mature.

What is different? Our authors point to three key changes. First, institutional investors are now resistant to investing in biotechnology because of the small size of the companies involved and because of their lack of liquidity. The influx of cash into the financial investment community has been so great that it has forced institutional investors to make increasingly large placements in order to manage their workflow. Because most biotechnology companies are too small to justify placements of $\$ 50$ million and up, they are no longer in the running for this financing. The drying up of this major source of biotechnology funding has been a serious blow.

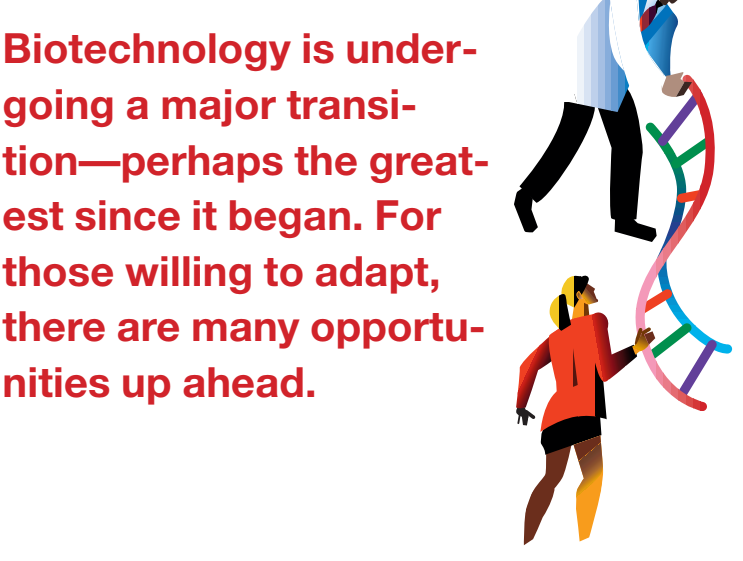

New business models

While all of our authors were confident about biotechnology's longterm future, the environmental factors described above suggested to some a need for new business plans representing a significant departure from present strategies. The alternative business models our authors propose demonstrate that, at least when it comes to finance, creativity is still alive and well in the industry. They range from creating a virtual company that is intimately involved in academic science to forming spinout companies around technologies that inventors don't have the time or

The second change is the effect of merger and acquisition (M\&A) activity on the pharmaceutical industry. As pharmaceutical companies undergo the M\&A process, they are redefining themselves in ways that make them more risk averse. In this new light, coordinating the activities of a dozen biotechnology collaborations is much less appealing than managing a relationship with a single company. As a result, pharmaceutical companies now tend to look to large biotechnology companies that can provide a complete range of solutions. Again, because only a limited number of biotechnology companies are capable of providing this type of "total" solution, there has been a slowdown in collaborative funding of the biotechnology industry.

Finally, the trend of "niche" biotechnology companies has turned a number of these niche goods and services into commodities. This has resulted in low-margin, high-volume businesses that most investors shun. Moreover, pharmaceutical companies are becoming resistant to the notion that a biotechnology company can service them and their competitors equally well by supplying them with the same technology. In this supplement, for example, Paul Herrling of Novartis discusses concerns about intellectual property rights, as well as concerns about the cost and feasibility of integrating a biotechnology company into Novartis' corporate culture. This is a warning to biotechnology that "big pharma" has regained the will to reinvent itself internally as "big biotech" if it cannot find what it needs on the market. money to develop.

Many authors do think that consolidation is the answer-at least for the short term. A merged company has a larger market cap that appeals to institutional investors and is able to offer pharmaceutical companies the kind of "one-stop shopping" that is currently in demand. The investment community sees this consolidation process as a way of eliminating the unnecessary duplication of effort required to fund lots of small companies in a particular niche, and as a way to make the new merged company to behave more like a business-forcing it to drop its "academic" projects and focus only on the most commercially promising ones. They believe that a leaner industry would be more effective in producing profitability for investors.

However, even advocates of consolidation agree that this would be a tough process to implement industry-wide, given the nature of the bioentrepreneurs who create these companies-highly competitive individuals who are absolutely committed to realizing their visions. More than one author pointed out that even if you could convince a bioentrepreneur that consolidation was a good idea, the ultimate resistance to implementation might come from the members of the bioentrepreneur's board of directors-who also come to have a passionate proprietary stake in the company they oversee.

We want to thank our authors for taking the time to share their insights. The lessons presented here should prove useful to all who are interested in helping to shape the next phase of biotechnology's growth and development. 